\title{
In-situ field emission characterisation of multi walled carbon nanotubes
}

\author{
R. C. Smith", J. D. Carey, D. C. Cox and S. R. P. Silva \\ Nano-Electronics Centre, Advanced Technology Institute, University of Surrey, Guildford, GU2
}

$7 X H, U K$.

The field emission (FE) properties of groups of carbon nanotubes (CNTs), an individual CNT, and from a single gated CNT are measured using nano-manipulators fitted within an SEM (scanning electron microscope). We investigate the effect of the anode locations at anode to emitter tip distances as low as $1 \mu \mathrm{m}$.

Nano-manipulators were installed within an SEM chamber, and sharpened STM probes with end radius of $5 \mu \mathrm{m}$ attached to the end of the two manipulators. The minimum step size achievable was $40 \mathrm{~nm}$, with the FE performed within the SEM chamber at a vacuum pressure in the range of $10^{-7}$ Torr. Figure 1 shows the 2 nano-manipulators within the SEM chamber. The sequence of images show one of the probes placed accurately above a single CNT.

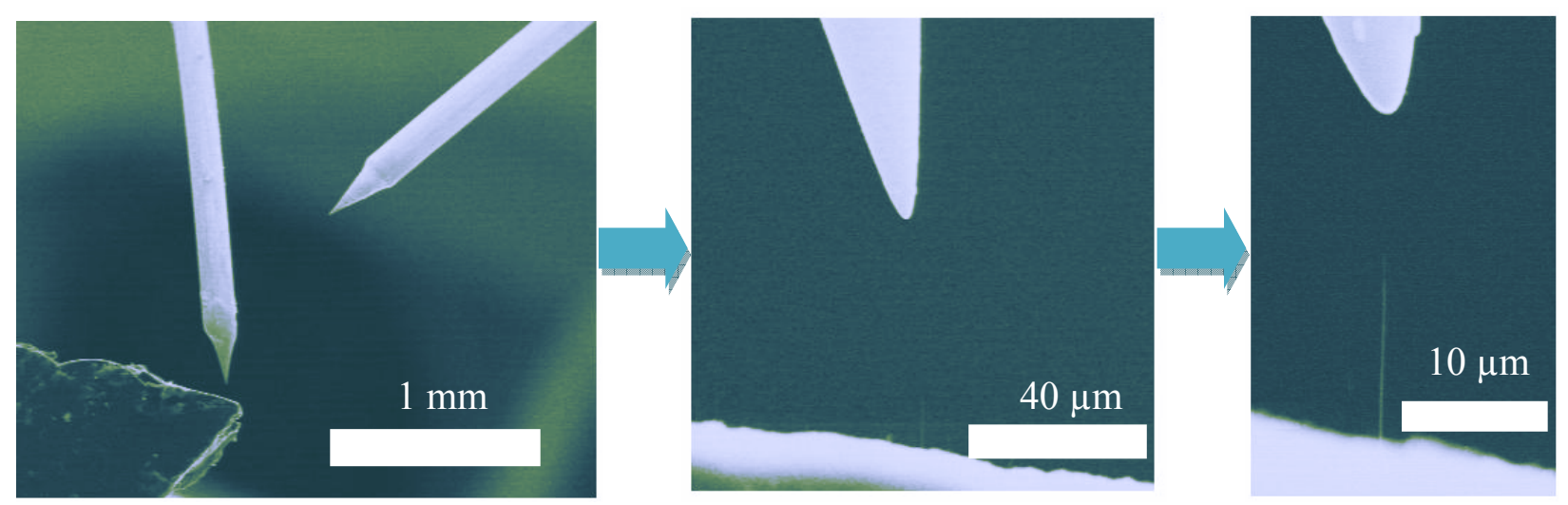

Figure 1. Series of three SEM images showing the two nano-manipulators within the vacuum chamber above a CNT-polymer composite sample (left), and positioned $10 \mu \mathrm{m}$ above an isolated CNT (right).

A group of multi walled CNT were attached randomly to the end of a carbon fibre, and their field emission characteristics recorded. It was noted that the position of the anode (which could be measured accurately within the SEM) played an important role in the performance of the emitter. Both threshold field and enhancement factors were affected only at low anode to emitter tip separations. To further our studies we performed similar emission experimentation on an isolated CNT. A CNT polystyrene polymer was physically broken to expose a CNT, and its edge scanned to find a suitable emitter. Field screening effects due to adjacent emitters were removed by our ability to selectively burn off, or pull out unwanted CNTs. During FE testing the electron beam was turned off as to not influence our results. 
It was proposed that the change in threshold field and enhancement factor could be explained by introducing an alternative definition for applied field. If the separation is taken as the anode to tip separation (D-h), rather than the anode to cathode separation (D), we see a decrease in enhancement factor as the anode approaches the CNT tip. This can be explained by the fact that at close anode proximity, the CNT cap to anode radius can be assumed to approximate a parallel plate, which would have an enhancement factor of 1 .
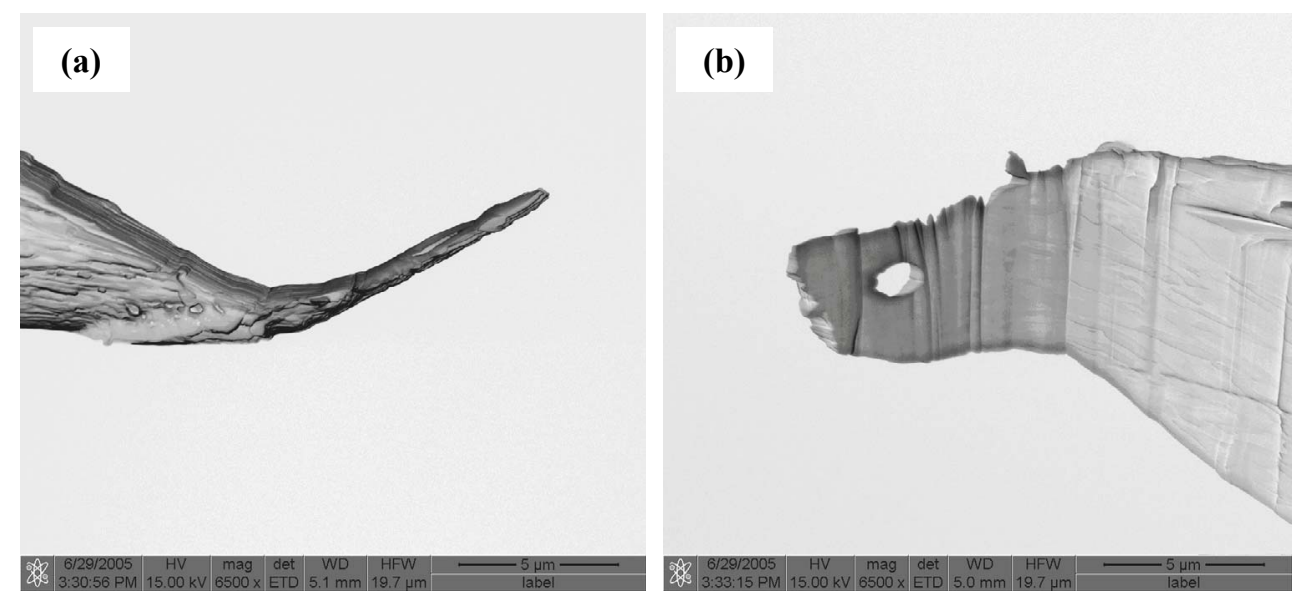

Figure 2. The gate electrode, fabricated by focused ion beam milling of a Tungsten STM probe.

Further to our in-situ investigations, we were able to perform three terminal field emission on an isolated CNT. A bespoke gate electrode was fabricated by focussed ion beam (FIB) milling (figure 2 (a and b)), and accurately positioned above the CNT. Our results show that the presence of the gate electrode can screen the performance for the CNT emitter by up to $66 \%$. Figure 3 shows the field emission characteristics for gate voltages ranging between 0 and 25 volts. The star points are the results when the gate electrode was moved out of the field of view.

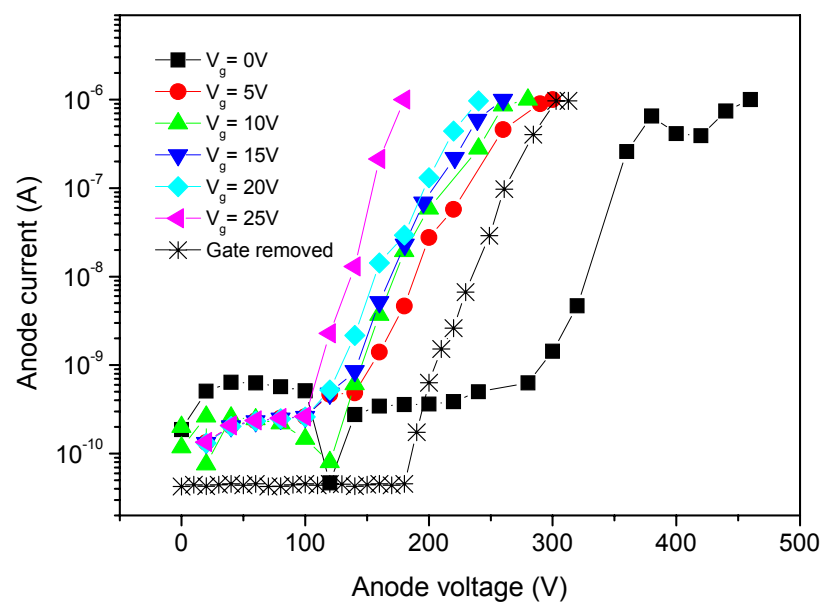

Figure 3. Field emission characteristics for the gated single CNT at gate voltages between $0-25 \mathrm{~V}$, and for the case when the gate was removed.

* Email: r.c.smith@eim.surrey.ac.uk 